\title{
Research Article \\ Enhance the Light Extraction Efficiency of QLED with Surface Micro-Nanostructure
}

\author{
Zilei Liu, Feng Li $\mathbb{D}$, Gaoxiang Huang, Jiahu Wei, Guangyu Jiang, and Yan Huang \\ Jiangxi Engineering Laboratory for Optoelectronics Testing Technology, Nanchang Hangkong University, Nanchang 330063, China
}

Correspondence should be addressed to Feng Li; lifengnchu@163.com

Received 14 August 2020; Revised 9 December 2020; Accepted 11 December 2020; Published 24 December 2020

Academic Editor: Filippo Giubileo

Copyright @ 2020 Zilei Liu et al. This is an open access article distributed under the Creative Commons Attribution License, which permits unrestricted use, distribution, and reproduction in any medium, provided the original work is properly cited.

\begin{abstract}
Quantum dot light-emitting diodes (QLEDs) are usually a flat multilayer structure. The luminous efficiency of QLEDs is limited because the light extraction is constrained owing to the substrate mode at the air/substrate interface, the waveguide mode at the conductive glass/organic interface, and the surface plasmon polaritons (SPPs) along the metal/organic interface. To improve the light extraction for the luminous efficiency of QLEDs, light transmission through the QLEDs coupled with three different surface micro-nanostructures is simulated by using a ray-tracing method. Furthermore, QLEDs (red, green, and blue emission) coupled with micro-nanostructures are also fabricated to investigate the influence of the micro-nanostructures on the luminous efficiency. Our simulation results show that more light escapes from the QLEDs coupled with surface micro-nanostructures than the control devices without micro-nanostructures. Our experimental results are consistent with the simulation results. By coupling with surface micro-nanostructures, the luminous efficiencies of the devices exhibited great improvement, and the EQE of $\mathrm{R} / \mathrm{G} / \mathrm{B}$ trichromatic reached $17.3 \%, 2.81 \%$, and $10 \%$, respectively, which are about 1 2 times higher than those of the control devices without any surface structure. Our results should make a contribution to building a strategy for improving the luminous efficiency of QLEDs.
\end{abstract}

\section{Introduction}

Since Colvin and his coworkers reported their pioneering researches on light-emitting diodes (LEDs) based on cadmium selenide nanocrystals in 1994 [1], employing semiconductor nanocrystals or quantum dots (QDs) as emitter species in LEDs has received extensive attention owing to their convenient solution-process ability and low-cost fabrication [2]. A typical QD-based LED (QLED) consists of a multilayer structure, which includes, generally in the following order, glass substrate/anode/hole injection layer/hole transfer layer/QDs/electron injection layer/cathode. Generally, the external quantum efficiency (EQE) of QLED is the product of radiative efficiency $\left(\eta_{\text {rad }}\right)$, excitation efficiency $\left(\eta_{\text {exc }}\right)$, and extraction efficiency $\left(\eta_{\text {ext }}\right)$. During the past twenty years, substantial hard work, including various synthesis strategies and surface modification for QDs, structure engineering, and material optimization for devices, has been proposed and carried out to enhance the EQE of QLEDs. Currently, QDs of the three primary colours (red, green, and blue) all possess high photoluminescence (PL) quantum yields (QYs) nearly or approaching $100 \%$. Even so, due to the dot-dot energy transfer, QDs in the film state show significant reduction in QYs as compared with those in the solution. The underlying mechanism of the interdot energy transfer is not clear. However, it is confirmed that the reduced dot-dot distance can lead to an increased energy transfer that may quench the emission [3]. Fortunately, ligands with a long chain and the thick shell of QDs keep the dot away from each other when forming a QD solid, which benefits the emission efficiency of the QD film [4-6]. Besides, for achieving high $\eta_{\text {rad }}$, unbalanced electron and hole injection inside the device is the main barrier, which has been relieved effectively by using hole transfer materials of high mobility or blocking the excess electron injection with an insulating organic layer. Furthermore, similar to the organic LEDs (OLEDs), the substrate mode at the air/substrate interface, waveguide mode at the indium tin oxide (ITO)/organic interface, and the surface plasmon polaritons (SPPs) along the metal/organic interface are the three main optical modes 


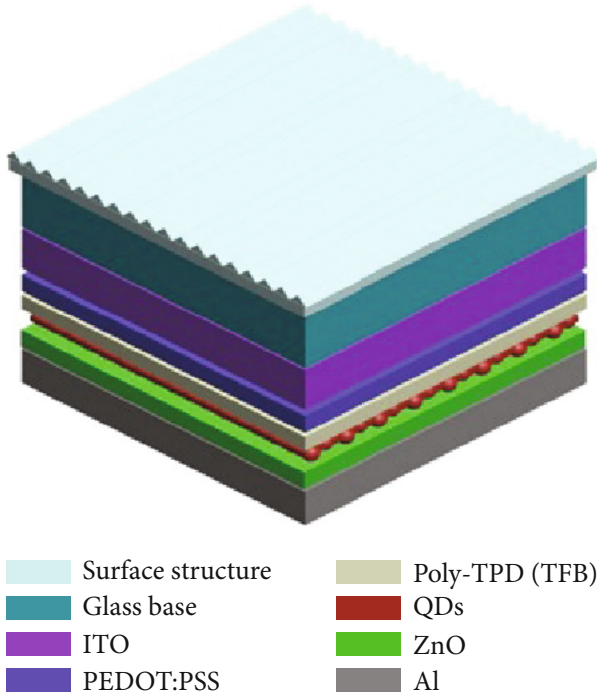

(a)

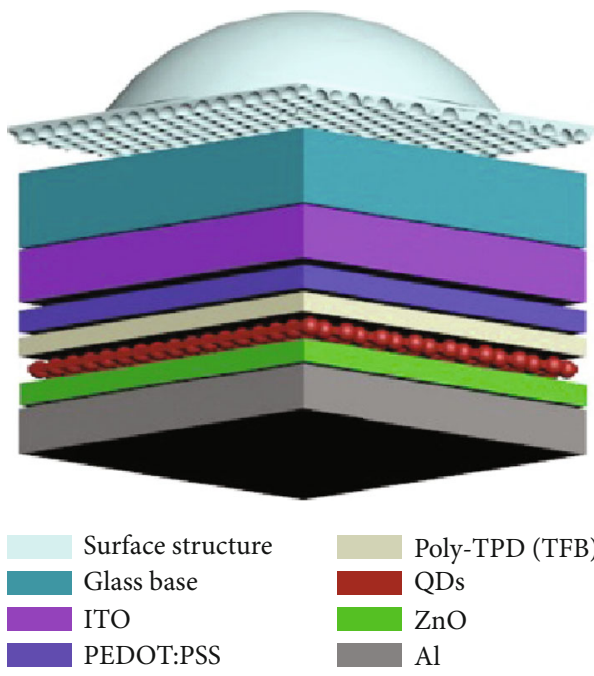

(c)
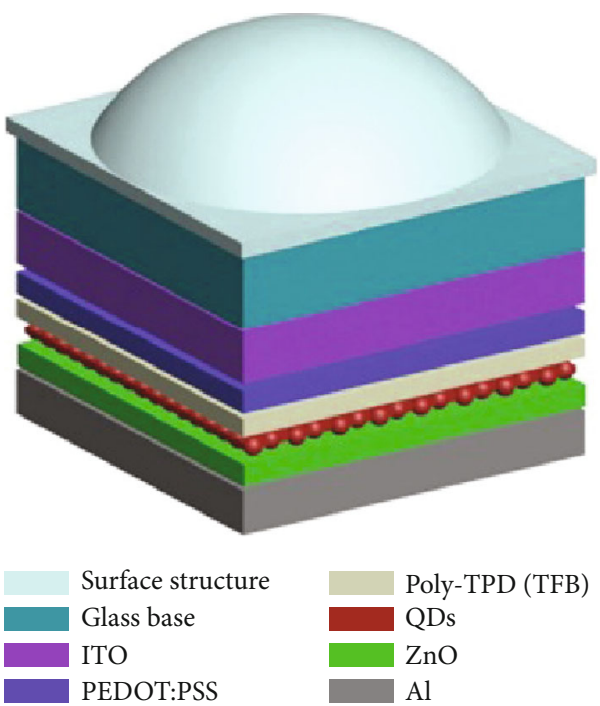

(b)

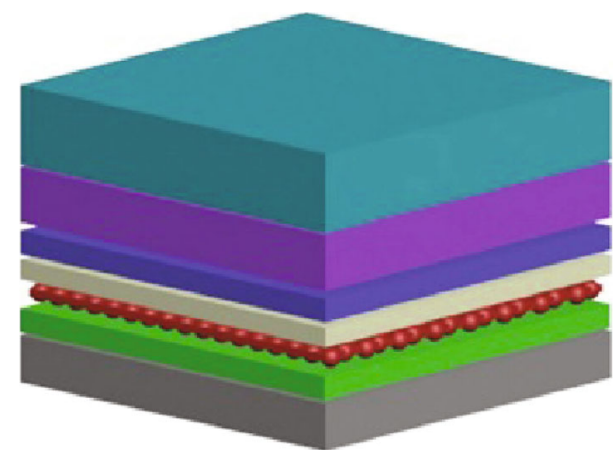

Surface structure

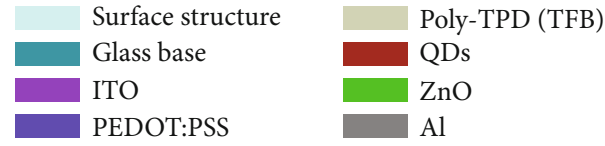

(d)

FIGURE 1: The models of QLEDs with surface micro-nanostructure: (a) QLED coupled with a microprism array (Model I), (b) QLED coupled with a flat convex lens (Model II), and (c) QLED coupled with a flat convex lens whose bottom is filled with a compound eye structure (Model III). (d) A control model without micro-nanostructure (Model IV).

that are harmful for the penetration of light through the multilayer structure, limiting $\eta_{\text {ext }}$ of the device $[7,8]$. Microstructures, including photonic crystals and gratings, have been employed to extract effectively the waveguide modes and SPPs inside OLEDs. By using a microlens array or light scattering layers, substrate modes can also be extracted significantly for OLEDs [9]. These methods have now been applied extensively in OLEDs to enhance their luminous efficiency. However, seldom in-depth investigations have been cast on the enhancement of $\eta_{\text {ext }}$ for QLEDs, and most researchers still try to improve the efficiency of luminescence by improving material properties and/or changing functional layers. Up to now, the champion EQEs for red, green, and blue QLEDs are 20.5\%, 21.0\%, and 19.8\%, respectively [1012]. Recently, optimized red InP-based QLED with EQE beyond $21.4 \%$ was reported [13]. Although the luminous efficiency of QLEDs has exhibited great progress, it still lags far behind that of OLEDs; besides material engineering and structure optimization, cooperation of micro-nanostructure may provide QLEDs with performance improvement in a short period of time.

Herein, we introduced three micro-nanostructures upon the light exit surface of QLEDs with a multilayer structure and carried out both simulation and experimental investigations on their influences on the luminous efficiency of the devices. For simulation investigation, the ray-tracing method was used to analyse the light transmission through the QLEDs with/without micro-nanostructures. The simulation results indicated that the microlens array is beneficial for the light extraction of QLEDs. Furthermore, for experimental studies, QLEDs with a typical structure (ITO/PEDOT:PSS/poly-TPD (TFB)/QDs/ZnO/Al) based on CdSe/CdS/ZnS 
TABLE 1: Parameters for the ray-tracing simulation.

\begin{tabular}{lcccccccc}
\hline & ITO & PEDOT:PSS & Poly-TPD (TFB) & QDs & ZnO & Al & Flat convex lens & Microprism array \\
\hline Thickness $(\mathrm{nm})$ & 180 & 40 & 50 & 40 & 30 & 70 & - & - \\
Refractive index & 1.8941 & 1.508 & 1.424 & 2.3631 & 1.6161 & 1.2623 & 1.492 & 1.492 \\
\hline
\end{tabular}

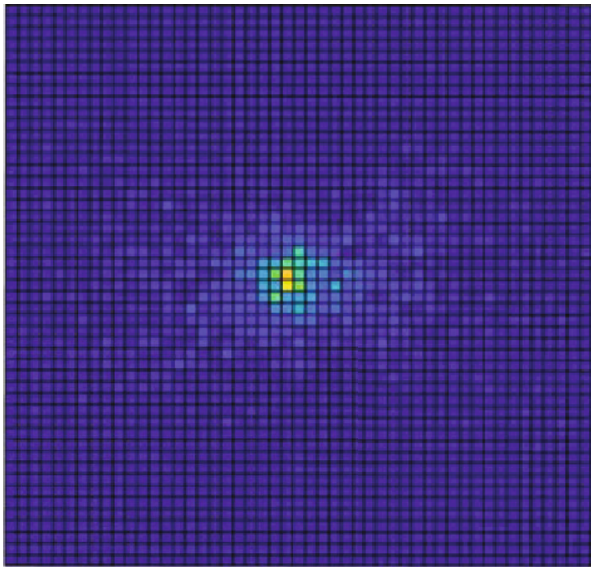

(a)

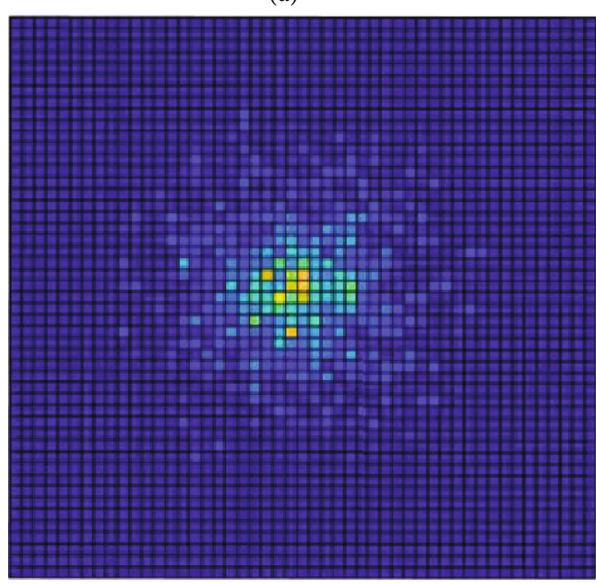

(c)

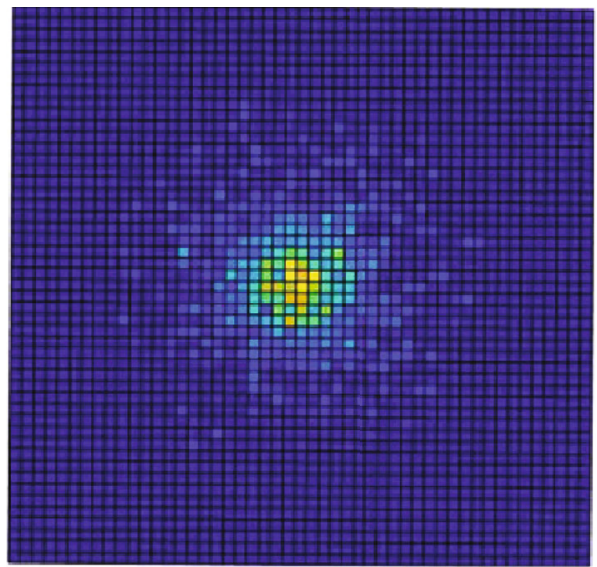

(b)

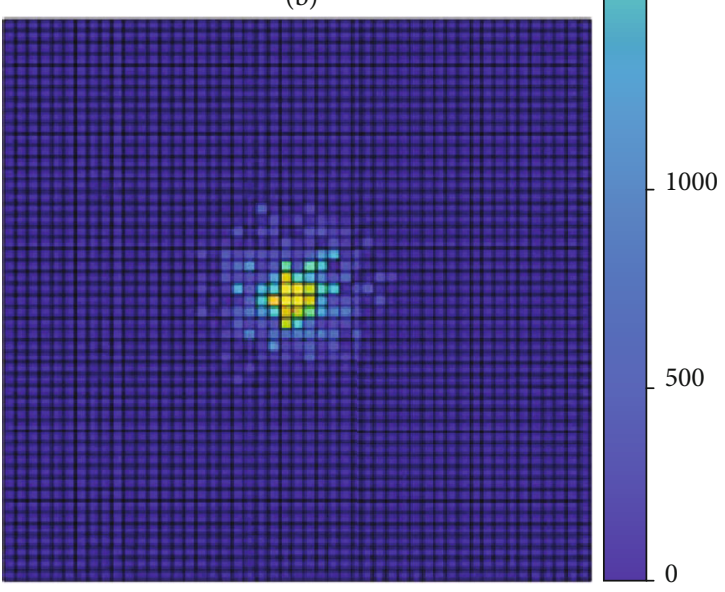

(d)

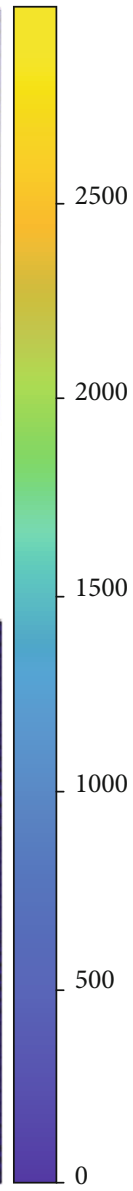

000

0

0 (1)

Figure 2: (a) Analysis of irradiance in Model I. (b) Analysis of irradiance in Model II. (c) Analysis of irradiance in Model III. (d) Analysis of irradiance in Model IV.

(red), CdSe/ZnS/ZnS (green), and CdZnS/ZnS (blue) QDs were fabricated. By integrating micro-nanostructures with the light exit surface, all the fabricated devices exhibited higher EQE than the control devices without micronanostructures. By coupling with a flat convex, the luminous efficiencies of the devices exhibited great improvement, and the EQE of R/G/B trichromatic reached $17.3 \%, 2.81 \%$, and $10 \%$, respectively. These values were about $1 \sim 2$ times higher than those of the control devices. Besides, when the light exit surface was coupled with a flat convex lens whose bottom is filled with a compound eye structure, the output lights show a more uniform angle distribution, despite the fact that the improvement of EQE was not as high as that obtained by using a simple flat convex lens. Our simulation and experimental results confirm that further $\mathrm{EQE}$ improvement should be possible through integrating the light exit surface with a surface micro-nanostructure.

\section{Simulation}

The ray-tracing method was used to demonstrate the effect of micro-nanostructures on the luminescence of QLEDs. The models for simulation are illustrated in Figure 1. The schematics for QLEDs coupled with a microprism array (Model I), a flat convex lens (Model II), and a flat convex lens whose bottom is filled with a compound eye structure (Model III) are given in Figures 1(a)-1(c), respectively. For comparison, a control model without any micro-nanostructure (Model IV) shown in Figure 1(d) is also investigated.

The device structure selected in the simulation is ITO/PEDOT:PSS/poly-TPD (TFB)/QDs/ZnO/Al. The thickness of ITO, poly-TPD (TFB), QDs, $\mathrm{ZnO}$, and $\mathrm{Al}$ was set to be $180 \mathrm{~nm}, 40 \mathrm{~nm}, 50 \mathrm{~nm}, 40 \mathrm{~nm}, 30 \mathrm{~nm}$, and $70 \mathrm{~nm}$, respectively. QDs with ZnS shell were used as the emitters in the simulation, and refractive indices of $\mathrm{ZnS}$ were employed as 
TABLE 2: Simulation results for different models.

\begin{tabular}{lcccc}
\hline & Model I & Model II & Model III & Model IV \\
\hline Number of output rays & 1825 & 3304 & 2709 & 1509 \\
Output luminous flux (W) & 0.047463 & 0.10593 & 0.094645 & 0.044141 \\
\hline
\end{tabular}
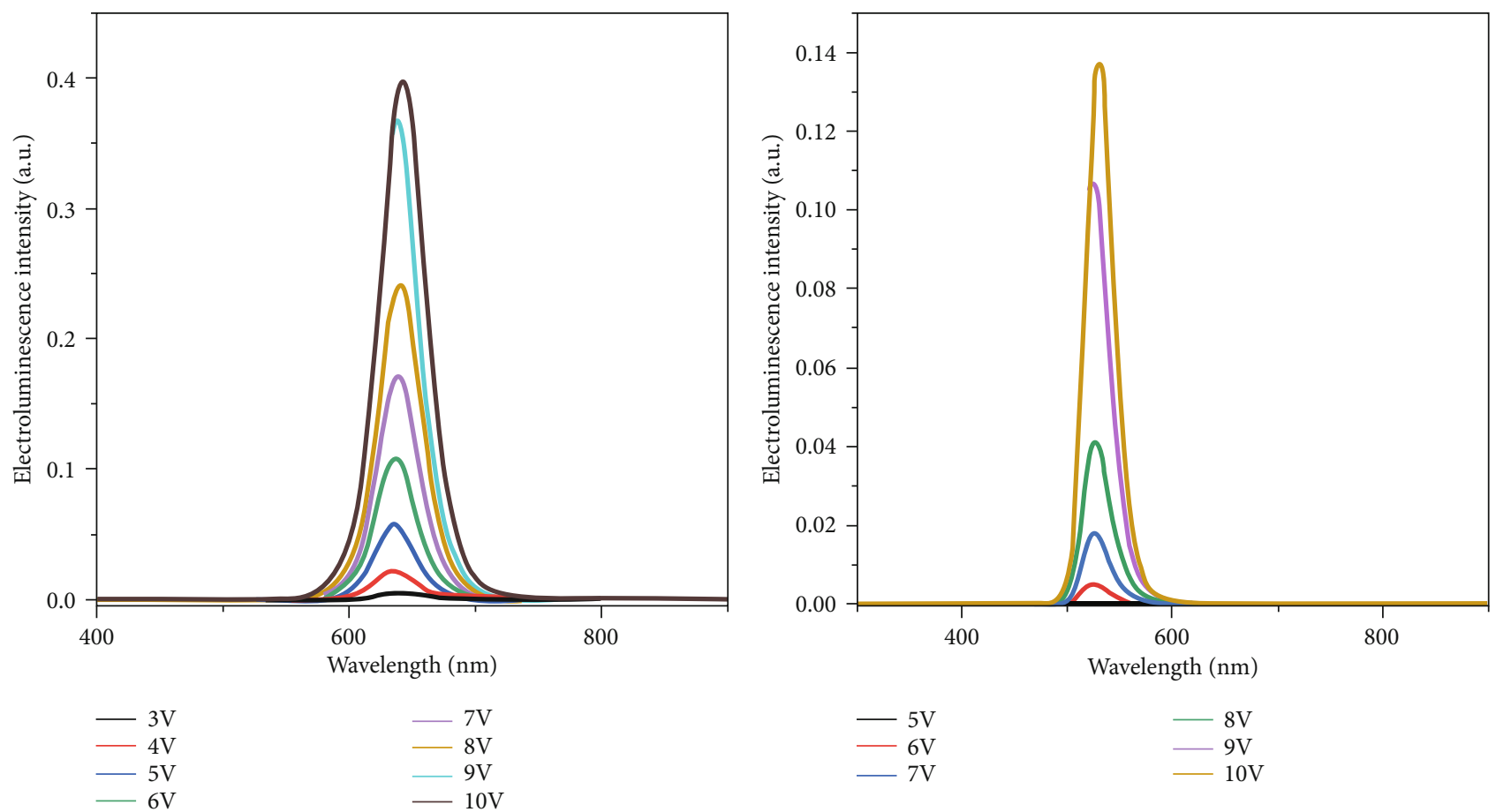

(a)

(b)
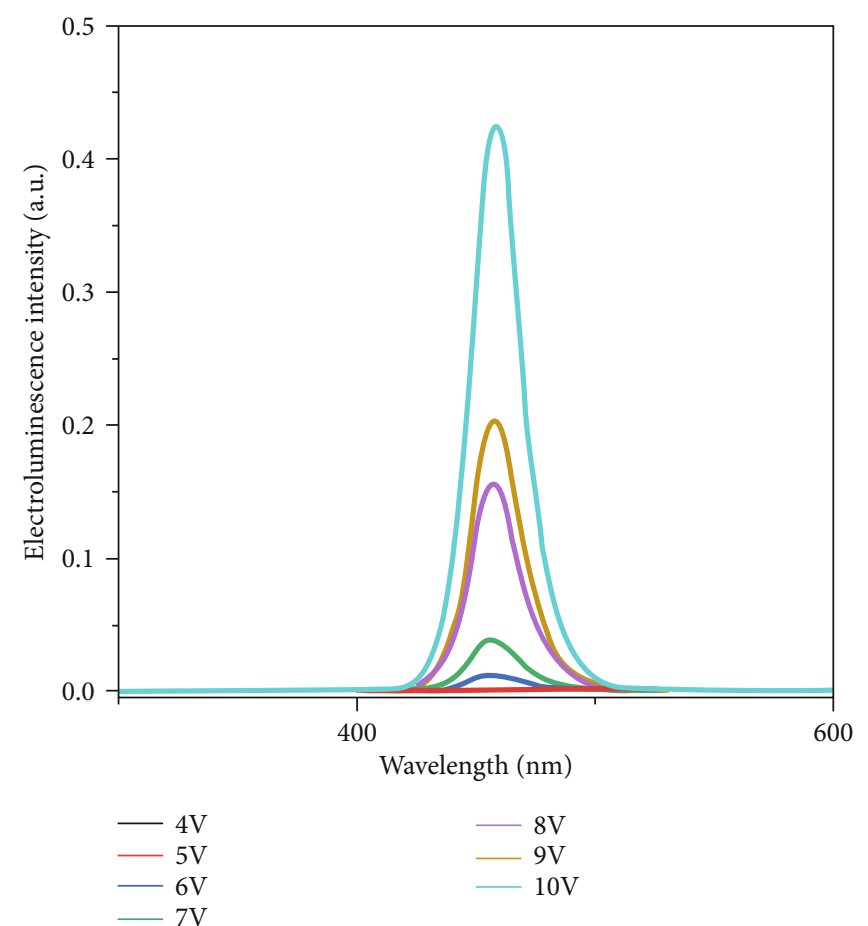

(c)

Figure 3: EL spectra of (a) red QLED based on CdSe/CdS/ZnS QDs, (b) green QLED based on CdSe/ZnS/ZnS QDs, and (c) blue QLED based on CdZnS/ZnS QDs. 


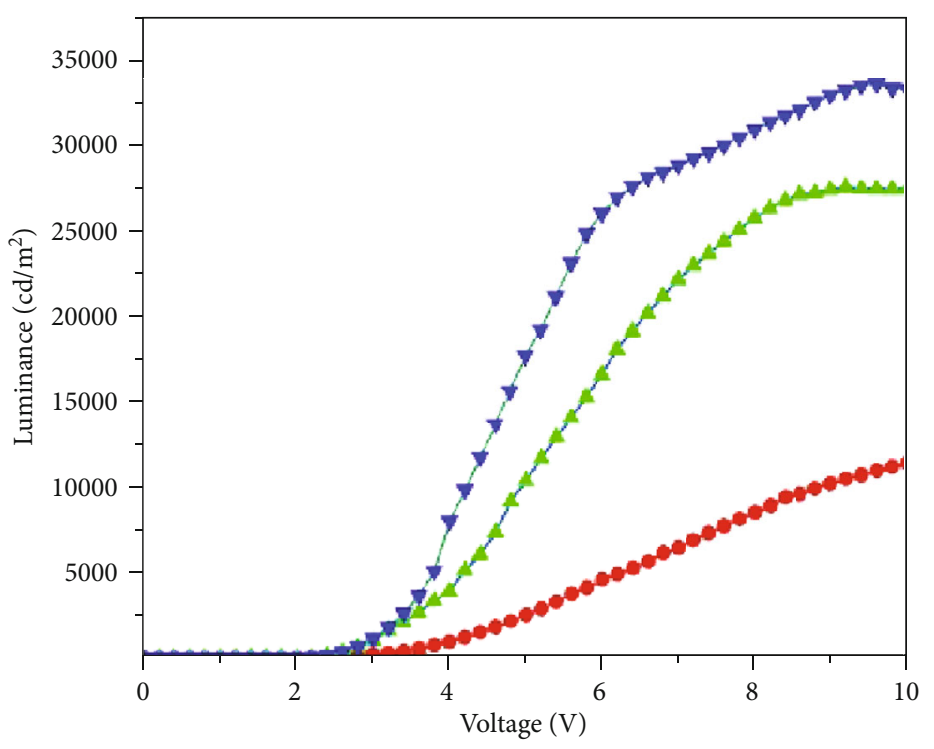

- No surface micro-nano structure

- Compound-eye structure plano-convex lens

$\checkmark$ Flat convex lens

(a)

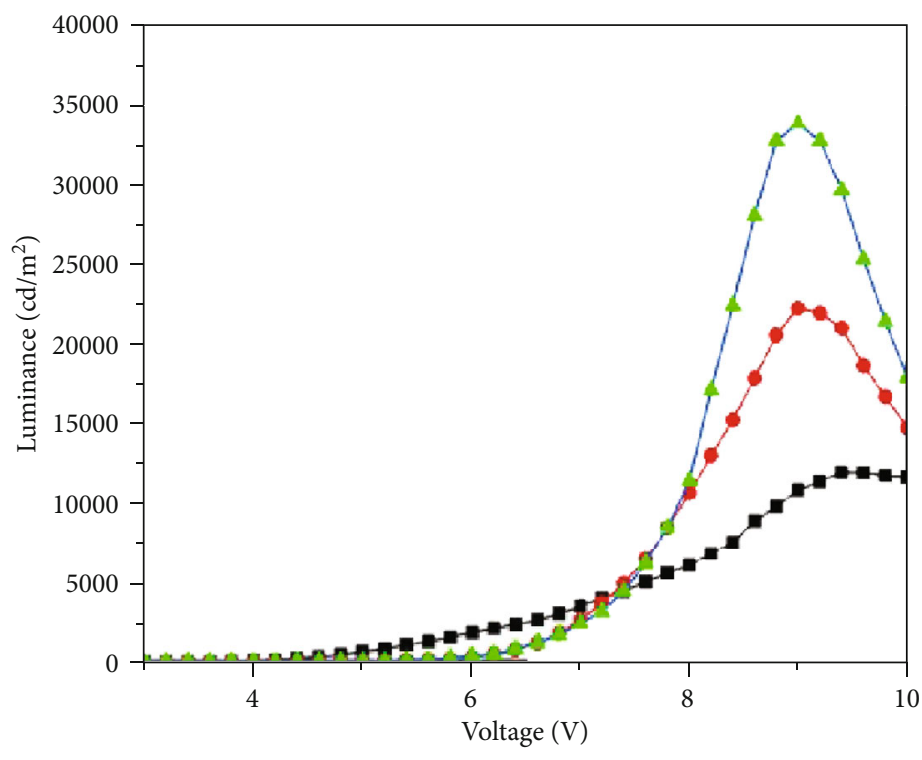

- No surface micro-nano structure

- Flat convex lens with compound-eye structure

- Flat convex lens

(b)

Figure 4: Continued. 


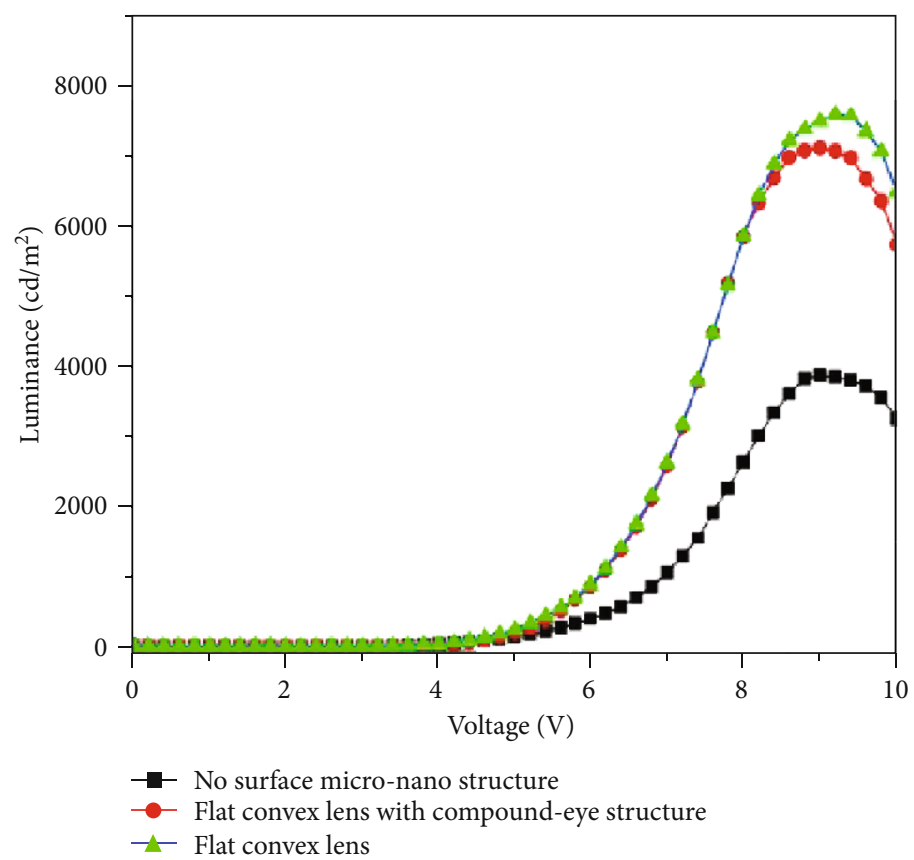

(c)

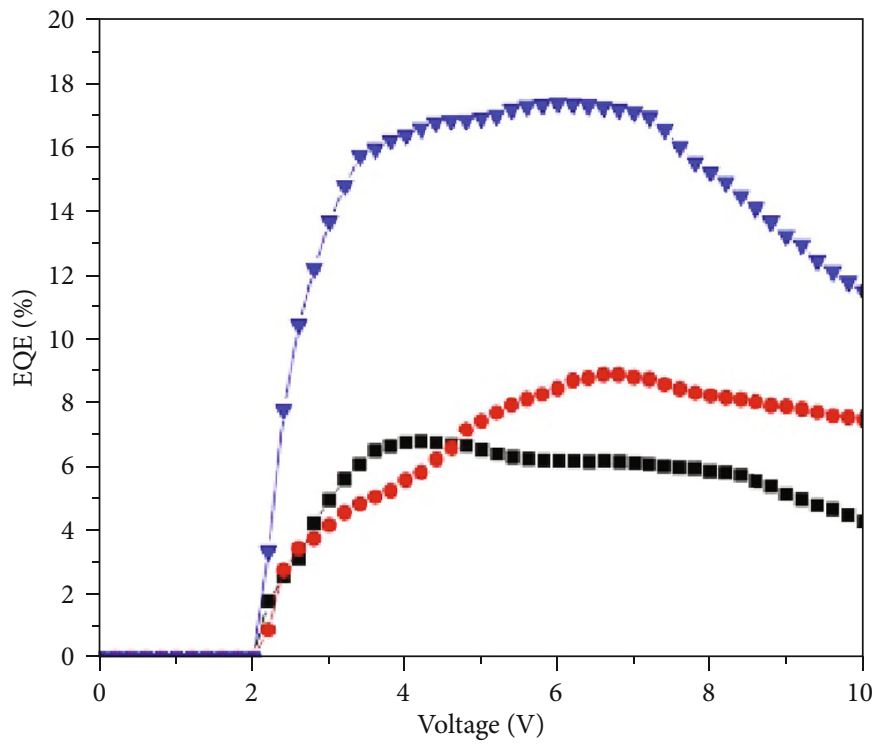

- No surface micro-nano structure

- Flat convex lens with compound-eye structure

$\checkmark$ - Flat convex lens

(d)

Figure 4: Continued. 


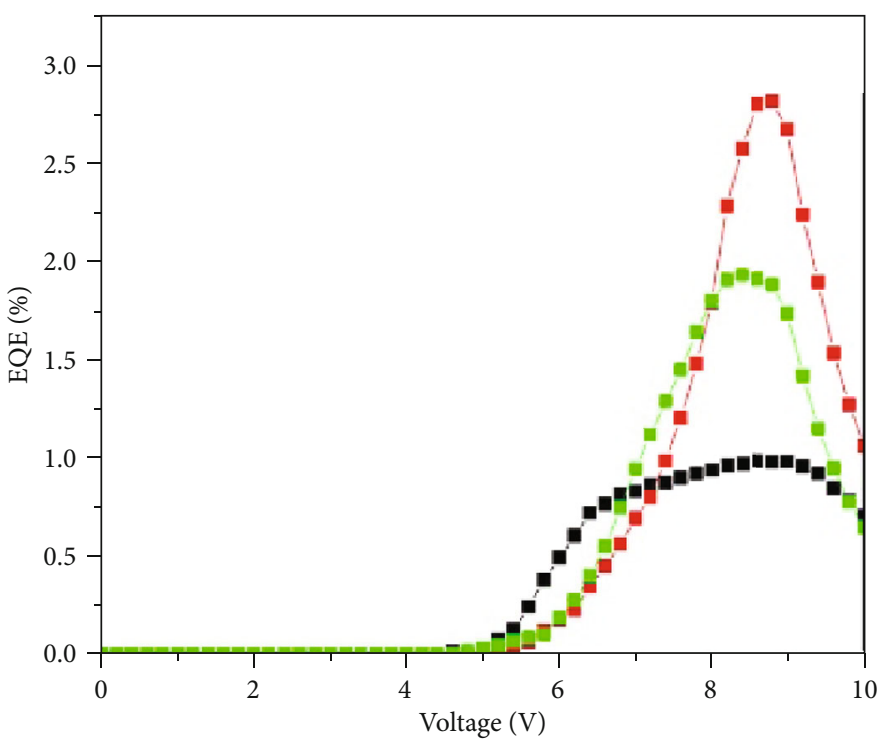

- No surface micro-nano structure

- Flat convex lens with compound-eye structure

- Flat convex lens

(e)

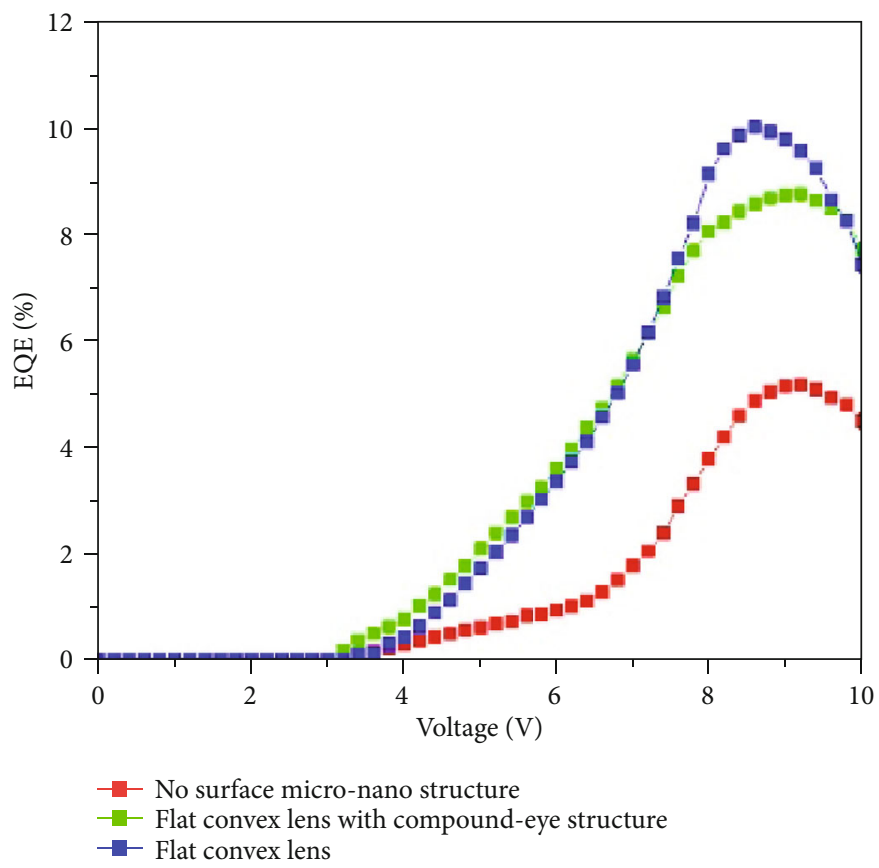

(f)

FIGURE 4: The luminance-voltage curves of QLEDs with three different light exit surfaces: (a) red QLED, (b) green QLED, and (c) blue QLED. The EQE-voltage curves of QLEDs with three different light-emitting surfaces: (d) red QLED, (e) green QLED, and (f) blue QLED.

that of the QD layer for convenience. Additionally, refractive indexes of polymer materials are dependent on the fabrication process and conditions. Hence, refractive indexes of ITO, PEDOT:PSS, QDs, $\mathrm{ZnO}$, and $\mathrm{Al}$ are adapted from reference [14], and they are 1.8941, 1.508, 2.3631, 1.6161, and 1.2623 , respectively. The refractive index of poly-TFB from reference [15] is used for both poly-TFB and polyTPD. All these parameters are summarized in Table 1. The microprism array on the surface of Model I consists of an equilateral prism with a length of $1.5 \mathrm{~mm}$ and a width of $0.01 \mathrm{~mm}$. The diameter of the flat convex Model II is $3 \mathrm{~mm}$, the focal length is $3.77 \mathrm{~mm}$, the central thickness is $2 \mathrm{~mm}$, and the edge thickness is $0.6 \mathrm{~mm}$. Model III has the same shape size in the simulation as Model II but equipped with a microlens array of micron levels at the bottom of the flat convex lens. Moreover, five thousand rays with total luminous power of $1 \mathrm{~W}$ were used to participate in the Monte-Carlo simulation. 
The simulation results are shown in Figures 2(a)-2(d), corresponding to the models in Figures 1(a)-1(d), respectively. It can be seen from Figure 2 that surface micronanostructures can reduce the internal total reflection and enhance the luminous efficiency obviously. It is calculated that the number of rays emitted outside from the QLED without an external coupling surface structure is only 1509, and the total luminous flux is $0.044141 \mathrm{~W}$. For Model I using the microprism array as the light exit surface, its simulation results are a little higher than those of the control model and with better light distribution. While for Models II and III, the number of rays emitted outside from QLEDs is 3304 and 2709, respectively, and the output luminous flux is 0.10593 and 0.094645 , respectively. These results exhibit distinguishable improvement compared to those of Models IV and I. The simulation results for all four models are summarized in Table 2.

Therefore, it is theoretically feasible to use surface micronanostructures to improve the luminescence performance of QLEDs.

\section{Materials and Methods}

3.1. Material. Poly(3,4-ethylenedioxythiophene)-poly(styrene sulfonate) (PEDOT:PSS), dimethyl sulfoxide (DMSO), tetramethyl hydroxide (TMAH), hexane (AR), ethanol $(\mathrm{AR})$, isopropanol (AR), acetone (AR), and chlorobenzene (98\%) were purchased from Sinopharm Chemical Reagent Co., Ltd. Zinc acetate $\left(\mathrm{Zn}\left(\mathrm{CH}_{3} \mathrm{CO}_{2}\right)_{3}\right.$, analytically pure (AR)), zinc acetate dihydrate $\left(\mathrm{Zn}\left(\mathrm{CH}_{3} \mathrm{CO}_{2}\right)_{3} \cdot 2 \mathrm{H}_{2} \mathrm{O}\right.$, AR, 99.0\%), dimethyl sulfoxide (99.7\%), 1-dodecanethiol (DDT, 98\%), and n-octane (99\%) were purchased from Macklin. Poly[bis(4-phenyl) (4-butylphenyl)amine] (poly-TPD) and poly[(9,9-dioctylfluorenyl-2,7-diyl)-co-(4,49-(N-(4-sec-butylphenyl)) diphenylamine)] (TFB) were purchased from American Dye Source. Indium tin oxide (ITO) glass substrates were purchased from Xiamen Weihua. CdSe/CdS/ZnS (red), $\mathrm{CdSe} / \mathrm{ZnS} / \mathrm{ZnS}$ (green), and CdZnS/ZnS (blue) QDs were purchased from Suzhou Xingshu. Flat convex lens ( $3 \mathrm{~mm}$ diameter) and flat convex lens whose bottom is filled with a compound eye structure ( $8 \mathrm{~mm}$ diameter) were purchased from Nanyang Jingliang Optoelectronic Technology. All reagents were used as received without further experimental purification.

\subsection{Fabrication of $R / G / B$ Trichromatic $Q L E D s$}

(1) Firstly, $5.5 \mathrm{mmol}$ of the anhydrous zinc acetate was dissolved fully in $30 \mathrm{ml}$ of dimethyl sulfoxide (DMSO) under magnetic stirring to prepare $\mathrm{ZnO}$ solution; $3 \mathrm{mmol}$ of tetramethyl hydroxide (TMAH) was dissolved fully in $10 \mathrm{ml}$ of absolute ethyl alcohol under magnetic stirring. Next, these two solutions were mixed slowly; then, the resulted $\mathrm{ZnO}$ particles were purified and dissolved in ethanol for further use. Subsequently, QDs were dissolved in n-hexane and then precipitated with ethanol. The purification processes were repeated for about three times. After that, QDs were dried and then dissolved in n-octane for further use. ITO glasses were cleaned ultrasoni-
TABLe 3: Luminance and EQE values of QLEDs.

\begin{tabular}{lccccc}
\hline Colour & Model & $\begin{array}{c}\text { Luminance } \\
\left(\mathrm{cd} / \mathrm{m}^{2}\right)\end{array}$ & $\begin{array}{c}\text { Increment } \\
(\%)\end{array}$ & $\begin{array}{c}\text { EQE } \\
(\%)\end{array}$ & $\begin{array}{c}\text { Increment } \\
(\%)\end{array}$ \\
\hline \multirow{4}{*}{ Red } & IV & 10708.75 & - & 6.7 & - \\
& III & 27430.00 & 156 & 8.9 & 32 \\
& II & 33465.00 & 212 & 17.3 & 158.2 \\
\multirow{6}{*}{ Green } & IV & 11295.50 & - & 1 & - \\
& III & 21801.25 & 93 & 1.9 & 90 \\
& II & 32575.00 & 183 & 2.81 & 183 \\
\multirow{6}{*}{ Blue } & VI & 3864.00 & - & 5.2 & - \\
& III & 7100.00 & 83 & 8.5 & 63.46 \\
& II & 7485.25 & 93 & 10 & 92.3 \\
\hline
\end{tabular}

cally with deionized water, acetone, and isopropanol successively, so that unstained substrates can be obtained for the fabrication of QLEDs.

For the fabrication of QLEDs, PEDOT:PSS, poly-TPD, QDs, and $\mathrm{ZnO}$ were spin coated on the ITO glass substrate in turn in the glove box filled with inertia gas. The thickness of these films was controlled by adjusting the solution concentration and the rotating speed. Finally, aluminium is deposited on the surface of the $\mathrm{ZnO}$ layer by using a vacuum evaporation method. Finally, the slide glass is packaged on the chip by UV-curing adhesive, and the fabrication of QLED is completed.

(2) The prepared QLEDs were wiped with a cotton stick dipped in ethanol. The planoconvex lens and the planoconvex lens with the compound eye structure are bonded to the light-emitting surface of the QLED by the UV-curing glue

\section{Results and Discussion}

To obtain high-efficiency QLEDs, the rotating speed, the rotating time, and the solution concentration were optimized. Moreover, poly-TPD was used as the hole transport layer for the red-emission device and poly-TFB for the green and the blue ones. The electroluminescent (EL) spectra of the fabricated QLEDs of different emission colours without surface modification are shown in Figure 3. It can be seen from Figure 3 that the emission peaks of the devices are $645 \mathrm{~nm}$, $525 \mathrm{~nm}$, and $460 \mathrm{~nm}$, respectively. However, with the increase of applied voltage, the EL spectra shift to the longer wavelength slightly, which may be caused by the Stark effect [16] This phenomenon of spectral shift is similar to that of QLEDs based on $\mathrm{CdSe} / \mathrm{CdS} / \mathrm{ZnS}, \mathrm{CdSe} / \mathrm{ZnS} / \mathrm{ZnS}$, and $\mathrm{CdZnS} / \mathrm{ZnS}$ QDs reported previously [17-19].

A flat convex lens (with and without a compound eye structure) was coupled upon the light exit surface of the QLEDs by using UV-curing glue. The compound eye structure of the micro-nanometer level is regular and uniform. Figure 4 shows the luminance properties of the QLEDs with and without surface micro-nanostructures. The luminance of all the QLEDs reached their corresponding maximum with 


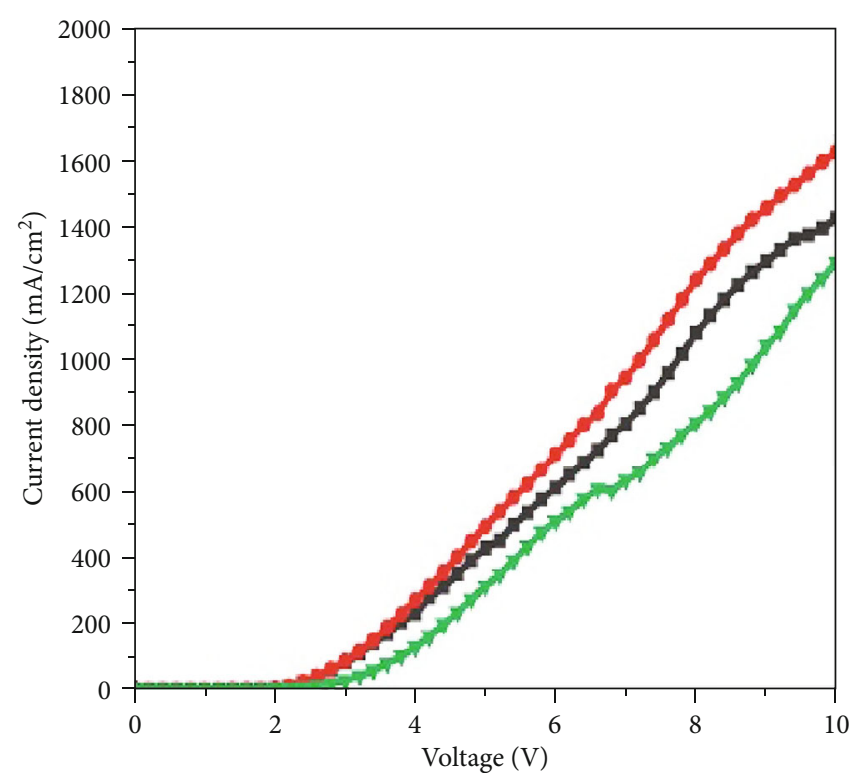

- No surface micro-nano structure

- Flat convex lens with compound-eye structure $\rightarrow$ Flat convex lens

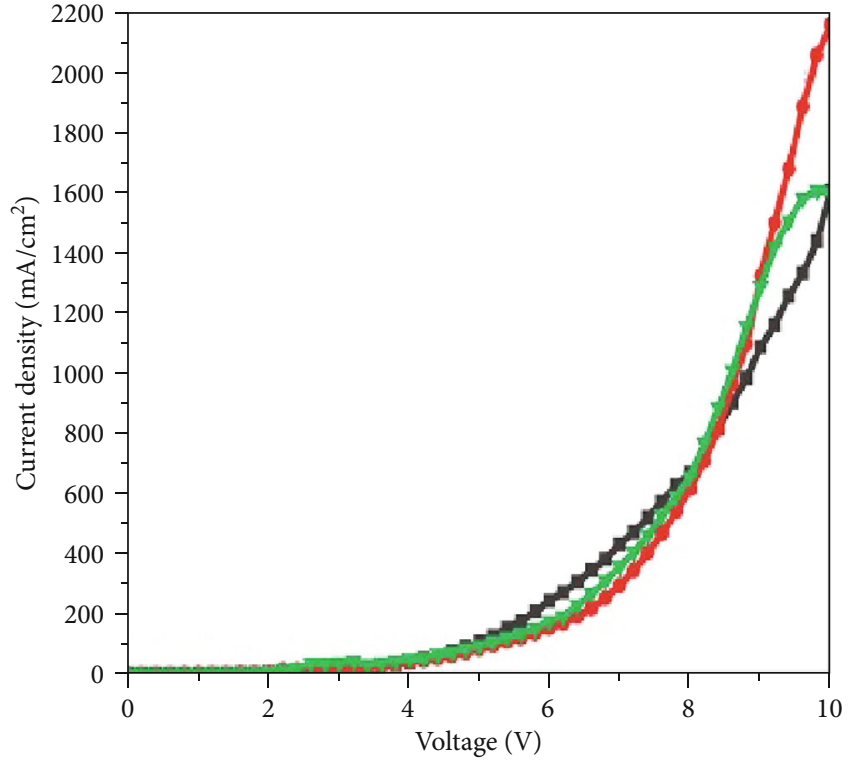

- No surface micro-nano structure

- - Compound-eye structure plano-convex lens

$\rightarrow$ Flat convex lens

(a)

(b)

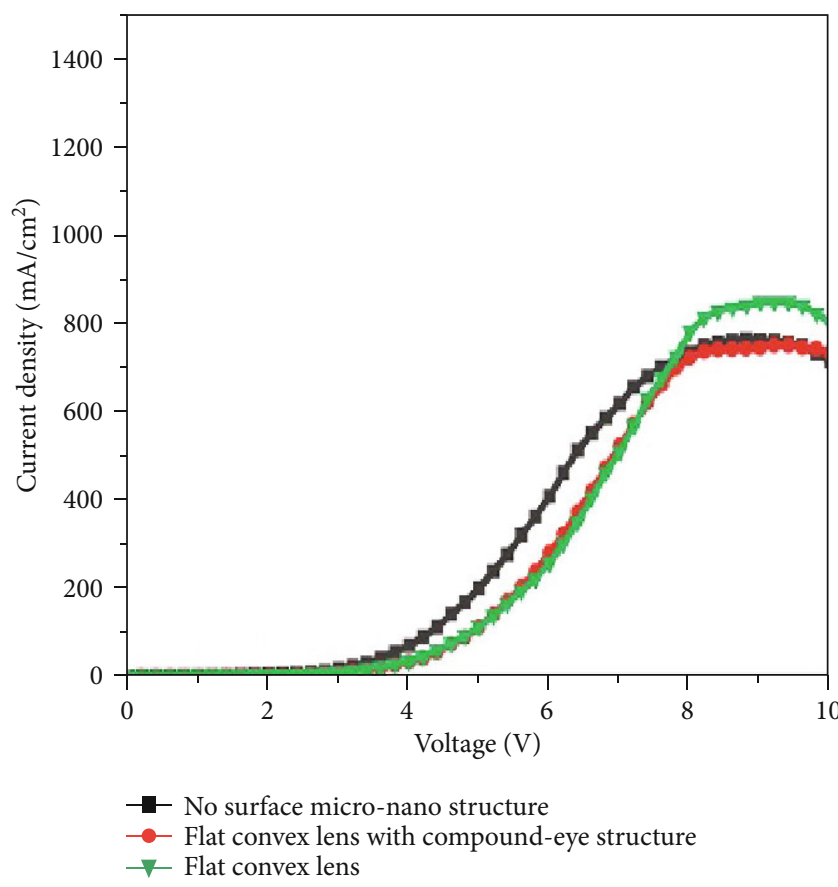

(c)

FIGURE 5: Current-voltage characteristics of QLEDs with three different light exit surfaces: (a) red, (b) green, and (c) blue.

an applied voltage of about $9 \mathrm{~V}$. However, the maximum values of the devices with a surface micro-nanostructure were higher obviously than that of the device without a surface micro-nanostructure. Moreover, with Model II, the maximum EQEs of R/G/B QLEDs are 17.3\%, 2.81\%, and $10 \%$, respectively. Additionally, with Model III, the maximum EQE values of the trichromatic QLEDs under the same driven voltage are $8.9 \%, 1.9 \%$, and $8.5 \%$, respec- tively. However, for the control device of Model IV, the corresponding maximum EQEs of the trichromatic QLEDs are $6.7 \%, 1 \%$, and $5.2 \%$, respectively. All these values are summarized in Table 3. It can be concluded that the luminous efficiency of QLEDs can be improved remarkably by coupling the light exit surface with a micro-nanostructure, which is in line with the simulation results in Section 2. 


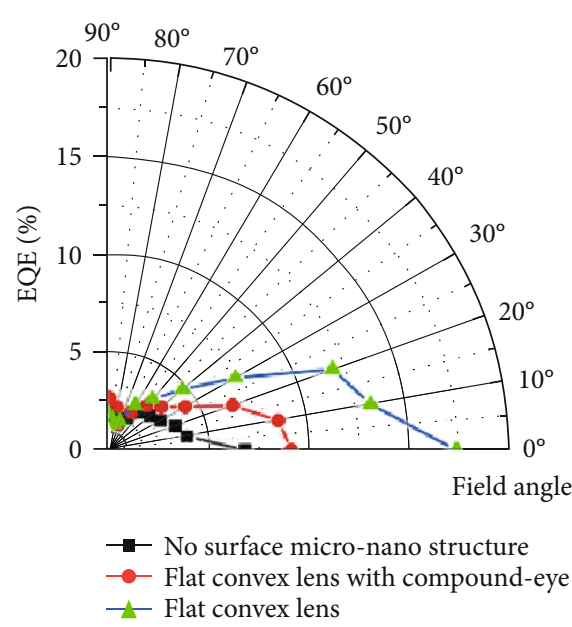

(a)

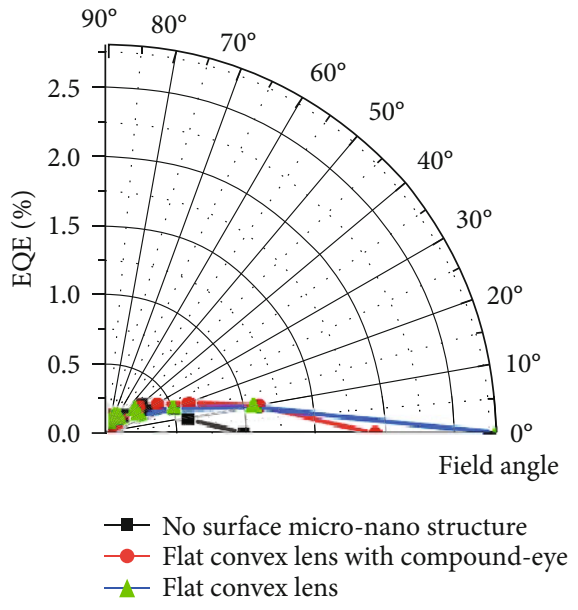

(b)

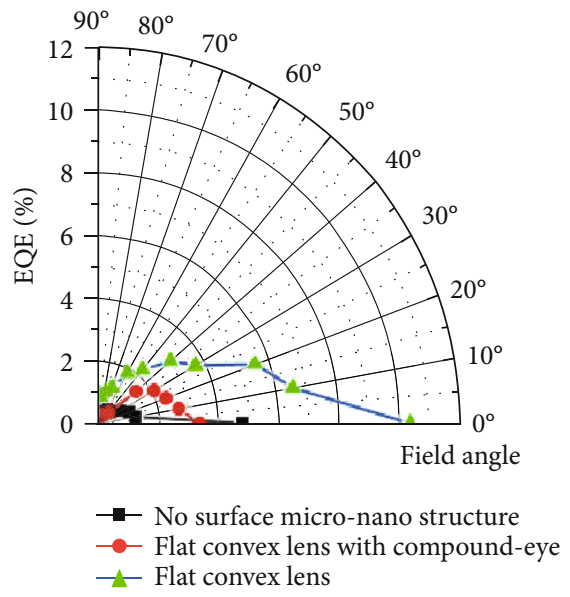

(c)

Figure 6: Angle distribution of the output light of QLEDs with three different light exit surfaces: (a) red, (b) green, and (c) blue.

Similarly, we consider the electrical changes of the device to help us understand the effects of the surface micronanostructures on the performance of the devices. The current density-voltage curves of R/G/B QLEDs with different surface structures are shown in Figure 5. It can be seen that the QLEDs exhibited analogous current-voltage characteristics whether they were or not coupled with micro-nanostructures. These results indicate that the introduction of micro-nanostructures upon the light exit surface of the QLEDs is electrically acceptable. In the view of luminance and EQE, a flat convex lens enables their great improvement, especially for the flat convex lens, which leads to doubling of the luminous efficiency of the red device. Hence, coupling the light exit surface with a micronanostructure can improve effectively the luminous performances of the devices with no adverse effect on the electrical properties. From the viewpoint of geometric optics, the convex lens surface structure is beneficial for the reduction of the total reflection effect and the exit of light. From the viewpoint of wave optics, the surface structure of the convex lens increases the escape cone of photons so that more photons can escape from the inside of devices.

Surface micro-nanostructures not only are helpful for improving the luminous efficiency of QLEDs but also change the light propagation trajectory. Here, the angle distribution of the output light of QLEDs was also measured and shown in Figure 6. It can be seen from Figure 6 that for the QLEDs coupled with micro-nanostructures, the propagation of the emitted light rays in the direction perpendicular to the light-emitting surface changes greatly compared to that of the control device. For all the control devices of different emission colours, EQEs decrease quickly with the increasing of the viewing angle from 0 (perpendicular to the light exit surface of the device) to 90 degrees. Furthermore, for the devices coupled with a micro-nanostructure, EQEs decrease more slowly with an increasing viewing angle. Thereinto, the output light from the QLEDs coupled with the flat convex lens exhibits better convergence effect, especially for the red and the blue emission devices. Due to the more photonic escape cones in the compound eye structure, the output light from the corresponding devices exhibits a relatively uniform angle distribution in a small angle range.

\section{Conclusions}

In this paper, we use the ray-tracing method to carry out the optical transmission through the QLEDs with/without 
surface micro-nanostructures. By using ray-tracing and irradiance analysis, the effect of the micro-nanostructure on the optical property of QLED is investigated. After that, QLEDs with the R/G/B three primary colours based on $\mathrm{CdSe} / \mathrm{CdS} / \mathrm{ZnS}, \mathrm{CdSe} / \mathrm{ZnS} / \mathrm{ZnS}$, and $\mathrm{CdZnS} / \mathrm{ZnS}$ core-shell QDs are successfully fabricated. To investigate the influence of the micro-nanostructure on the luminous properties of QLEDs, the flat convex lens and the flat convex lens with a compound eye structure are coupled upon the light exit surface of QLEDs. Compared with the control QLED without a micro-nanostructure, the EQE of QLEDs coupled with micro-nanostructures is improved obviously without changing the EL spectrum. The maximum EQE of the R/G/B three primary colours of the QLEDs coupled with the flat convex lens reaches $17.3 \%, 2.81 \%$, and $10 \%$, respectively, which are increased by $158.2 \%, 183 \%$, and $92.3 \%$, compared with those of the control devices, $6.7 \%, 1 \%$, and $5.2 \%$, respectively. Furthermore, the maximum EQE of the QLEDs with a compound eye structure of $8.9 \%, 1.9 \%$, and $8.5 \%$ for $\mathrm{R} / \mathrm{G} / \mathrm{B}$ emission colour, respectively, also exhibits obvious enhancement compared with those of the control devices. Additionally, although the compound eye structure flat convex lens exhibits inferior light extraction than the flat convex lens, the output light from the corresponding device shows a better angle distribution in a small angle range. Our results indicate that the introduction of the micro-nanostructure upon the light exit surface of the QLEDs is helpful for improving the luminous properties of the devices and can make a contribution to accelerate the application of QLEDs in the fields of illumination and display.

\section{Data Availability}

The data that support the findings of this study are available from the corresponding author upon reasonable request.

\section{Conflicts of Interest}

The authors declare that there is no conflict of interest regarding the publication of this paper.

\section{Acknowledgments}

The authors gratefully acknowledge the financial support from the Natural Science Foundation of China (62005113 and 11864026), the Natural Science Foundation of Jiangxi Province (20202ACBL202004 and 20192BBF60001), and the Science and Technology Project of Education Department of Jiangxi, China (GJJ180536).

\section{References}

[1] V. L. Colvin, M. C. Schlamp, and A. P. Alivisatos, "Light-emitting diodes made from cadmium selenide nanocrystals and a semiconducting polymer," Nature, vol. 370, no. 6488, pp. 354-357, 1994.

[2] X. Jin, K. Xie, T. Zhang et al., "Cation exchange assisted synthesis of $\mathrm{ZnCdSe} / \mathrm{ZnSe}$ quantum dots with narrow emission line widths and near-unity photoluminescence quantum yields," Chemical Communications, vol. 56, no. 45, pp. 6130 6133, 2020.

[3] G. H. Carey, L. Levina, R. Comin, O. Voznyy, and E. H. Sargent, "Record charge carrier diffusion length in colloidal quantum dot solids via mutual dot-to-dot surface passivation," Advanced Materials, vol. 27, no. 21, pp. 3325-3330, 2015.

[4] I.-S. Liu, H.-H. Lo, C.-T. Chien et al., "Enhancing photoluminescence quenching and photoelectric properties of CdSe quantum dots with hole accepting ligands," Journal of Materials Chemistry, vol. 18, no. 6, p. 675, 2008.

[5] A. H. Ip, S. M. Thon, S. Hoogland et al., "Hybrid passivated colloidal quantum dot solids," Nature Nanotechnology, vol. 7, no. 9, pp. 577-582, 2012.

[6] J. Lim, B. G. Jeong, M. Park et al., "Influence of shell thickness on the performance of light-emitting devices based on $\mathrm{CdSe} / \mathrm{Zn}_{1-\mathrm{X}} \mathrm{Cd}_{\mathrm{X}} \mathrm{S}$ core/shell heterostructured quantum dots," Advanced Materials, vol. 26, no. 47, pp. 8034-8040, 2014.

[7] Y. Luo, L. Wang, Y. Ding, L. Li, and J. Shi, "High lightextracting efficiency for OLED directly fabricated on doubleside nanotextured silica substrate," Optics Letters, vol. 38, no. 14, 2013.

[8] J. Hauss, T. Bocksrocker, B. Riedel, U. Lemmer, and M. Gerken, "On the interplay of waveguide modes and leaky modes in corrugated OLEDs," Optics Express, vol. 19, pp. A851-A858, 2011.

[9] S. Zhang, G. A. Turnbull, and I. D. W. Samuel, "Enhancing the emission directionality of organic light-emitting diodes by using photonic microstructures," Applied Physics Letters, vol. 103, no. 21, 2013.

[10] X. Dai, Z. Zhang, Y. Jin et al., "Solution-processed, highperformance light-emitting diodes based on quantum dots," Nature, vol. 515, no. 7525, pp. 96-99, 2014.

[11] J. R. Manders, L. Qian, A. Titov et al., "High efficiency and ultra-wide color gamut quantum dot LEDs for next generation displays," Journal of the Society for Information Display, vol. 23, no. 11, pp. 523-528, 2015.

[12] L. Wang, J. Lin, Y. Hu et al., "Blue quantum dot light-emitting diodes with high electroluminescent efficiency," ACS Applied Materials \& Interfaces, vol. 9, no. 44, pp. 38755-38760, 2017.

[13] Y.-H. Won, O. Cho, T. Kim et al., "Highly efficient and stable InP/ZnSe/ZnS quantum dot light-emitting diodes," Nature, vol. 575, no. 7784, pp. 634-638, 2019.

[14] https://www.horiba.com/fileadmin/uploads/Scientific/ Documents/TFilm/AR_SE02.pdf.

[15] http://add.lanxess.com/fileadmin/product-import/tributyl_ phosphate_tbp_en_rcr.pdf.

[16] J.-M. Caruge, J. E. Halpert, V. Bulović, and M. G. Bawendi, "NiO as an inorganic hole-transporting layer in quantum-dot light-emitting devices," Nano Letters, vol. 6, pp. 2991-2994, 2006.

[17] Q. Zhang, X. Gu, Z. Chen et al., "Enhancing extraction efficiency of quantum dot light-emitting diodes by surface engineering," Optics Express, vol. 25, no. 15, pp. 17683-17694, 2017.

[18] H.-T. Vu, C.-Y. Huang, H.-C. Yu, and Y.-K. Su, "Ultrathin PVK charge control layer for advanced manipulation of efficient giant CdSe@ZnS/ZnS quantum dot light-emitting diodes," Organic Electronics, vol. 63, pp. 349-354, 2018.

[19] Q. Lin, L. Wang, Z. Li et al., "Nonblinking quantum-dot-based blue light-emitting diodes with high efficiency and a balanced charge-injection process," ACS Photonics, vol. 5, no. 3, pp. 939-946, 2017. 